\title{
SIMULTANEOUS SELECTION FOR DOMINANT AND RECESSIVE MUTANTS
}

\author{
J. W. JAMES
}

School of Wool Technology, University of New South Woles, Kensington, Australia

Received 12.x.64

Only rarely can the course of evolution be followed readily in natural populations, so that considerable interest at taches to the case of industrial melanism in moths, where the process is well documented. In discussing industrial melanism, Smith (1958) wrote: "why is it that where melanics have established themselves as the most frequent variety in any area, they have proved to be due to dominant genes? In a number of species melanic varieties due to recessive genes are known, but in no case have they become common in nature." He then pointed out that for an initially rare gene selection is much more effective if the gene is dominant than if it is recessive, and since the spread of industrial melanism has been very rapid, it must have been the dominant genes which were involved.

In considering simultaneous selection for dominant and recessive mutants, however, two further points must be noted. The first is that if the mutant had been previously unfavourable and maintained at a low frequency by recurrent mutation, it will be proportionally more common if recessive than if dominant. If $q_{0}$ is the initial frequency of a recessive mutant, the initial frequency of a similar dominant mutant would be about $q_{0}^{2}$, other things being equal. It is necessary to consider to what extent, if any, this may compensate for the greater effectiveness of selection for the dominant gene. The second point is that when both genes are present in the same population, the selective pressure on each gene depends upon the frequency of the other.

This note deals with simultaneous selection for similar dominant and recessive mutants, taking into account both points mentioned above. There are two cases to be considered; that in which the dominant and recessive mutants occur at different loci, and that in which both occur at the same locus.

\section{TWO $\mathrm{LOCl}$}

Suppose a dominant melanic mutant occurs with frequency $p$ at locus $\mathbf{A}$ and a recessive melanic mutant occurs with frequency $q$ at locus $B$. The pale form has a relative selective disadvantage $s$ with respect to the melanic form. At the A locus only the non-mutant homozygote gives rise to pale forms, and under random mating a fraction $q^{2}$ of these will be melanic through the effects of the $B$ locus, so selection at this locus is selection against a recessive with selective disadvantage $s\left(\mathrm{r}-q^{2}\right)$ whence it is easily shown that

$$
\frac{d p}{d t}=s p(1-p)^{2}\left(1-q^{2}\right)
$$

Similarly at the B locus the selective advantage of the mutant homozygote is $s(1-p)^{2}$, whence

$$
\frac{d q}{d t}=s q^{2}(\mathrm{I}-q)(\mathrm{I}-p)^{2}
$$


From (I) and (2)

$$
\frac{d q}{d p}=\frac{q^{2}}{p(\mathrm{I}+q)}
$$

and thence it follows that

$$
\ln \left(\frac{q}{p}\right)-\frac{\mathrm{I}}{q}=\ln \left(\frac{q_{0}}{p_{0}}\right)-\frac{\mathrm{I}}{q_{0}} .
$$

where ln denotes the natural logarithm and $p_{0}, q_{0}$, are initial gene frequencies. The interesting point is the value of $q^{*}$, the frequency of the recessive mutant when the melanic has established itself as the predominant variety. At this stage $p=$ I nearly enough and so

$$
\ln \left(q^{*}\right)-\frac{\mathrm{I}}{q^{*}}=\ln \left(\frac{q_{0}}{p_{0}}\right)-\frac{\mathrm{I}}{q_{0}} .
$$

Thus $q^{*}$ depends on the actual value of $q_{0}$ and the relative magnitudes of $p_{0}$ and $q_{0}$. If $p_{0}$ is of order of magnitude $q_{0}$, then approximately

$$
\frac{\mathrm{I}}{q^{*}}-\ln q^{*}=\frac{\mathrm{I}}{q_{0}}+\ln q_{0}
$$

If $q_{0}$ is small $q^{*}$ will be very little larger. For example if $q_{0}=0.0 \mathrm{I}$, $p_{0}=0.001$ then $q^{*} \approx 0.011$.

\section{ONE LOCUS}

Suppose alleles $\mathrm{A}, a, \alpha$ occur at a locus, that $\mathrm{A}$ is dominant to both $a$ and $\alpha$ and that $\alpha$ is recessive to $a$. If $\mathrm{A}$ and $\alpha$ are melanic mutants, then pale forms arise only from genotypes $a a$ and $a \alpha$, and these have a relative selective disadvantage $s$. If $p, q$ are the frequencies of $\mathrm{A}, \alpha$ respectively, it is easily shown that under random mating

$$
\begin{aligned}
& \frac{d p}{d t}=s p(\mathrm{I}-q-p)(q-p+\mathrm{I}) \\
& \frac{d q}{d t}=s q(\mathrm{I}-q-p)(q-p) .
\end{aligned}
$$

It is interesting to note that the recessive allele $\alpha$ increases in frequency so long as it is more common than $\mathrm{A}$, but that if it is less common than $\mathrm{A}$ its frequency decreases. In particular, if $\alpha$ ever attains a frequency of one-half it will never be eliminated by subsequent selection. Now from (7) and (8)

$$
\frac{d q}{d p}=\frac{q(q-p)}{p(\mathrm{r}+q-p)}
$$

and hence

$$
\frac{\mathrm{I}-p}{q}+\ln \left(\begin{array}{l}
p \\
q
\end{array}\right)=\frac{\mathrm{I}-p_{0}}{q_{0}}+\ln \left(\frac{p_{0}}{q_{0}}\right)
$$

where $p_{0}, q_{0}$ are initial frequencies of $\mathrm{A}, \alpha$, respectively. Again it is of interest to ask whether $q^{*}$, the frequency of $\alpha$ when the melanic form is 
predominant, will be appreciable. Since the allele $a$ will be eliminated, $p^{*}=1-q^{*}$ and on making this substitution in (10)

$$
\ln \left(\frac{1-q^{*}}{q^{*}}\right)=\frac{1-p_{0}-q_{0}}{q_{0}}+\ln \left(\frac{p_{0}}{q_{0}}\right) .
$$

If both $p_{0}$ and $q_{0}$ are small, the right hand side of (I I) will be large and $q^{*}$ therefore small. For example if $q_{0}=0.01$ and $p_{0}=0.0001$, then $\ln \left(\frac{1-q^{*}}{q^{*}}\right) \approx 94.4$ and $q^{*}$ is of order of magnitude $10^{-40}$. Thus the allele will be eliminated.

\section{DISCUSSION}

It is clear that taking account of the two points mentioned does not have any appreciable influence on the conclusions which are drawn. Neither factor exerts sufficient influence to offset the greater effectiveness of selection for the dominant mutant.

One interesting difference between the one locus and two loci cases did arise. With two loci the recessive mutant increased in frequency at all stages, though of course, very slowly. With only one locus involved the recessive is favoured by selection in the early stages, but once the dominant mutant exceeds it in frequency, selection operates to reduce the frequency of the recessive and eventually to eliminate the recessive allele from the population.

However, although the two cases differ in principle, the end result is that in both cases the recessive mutant never reaches an appreciable frequency.

\section{REFERENCE}

sмiтh, J. M. 1958. The Theory of Evolution. Penguin Books. London. 